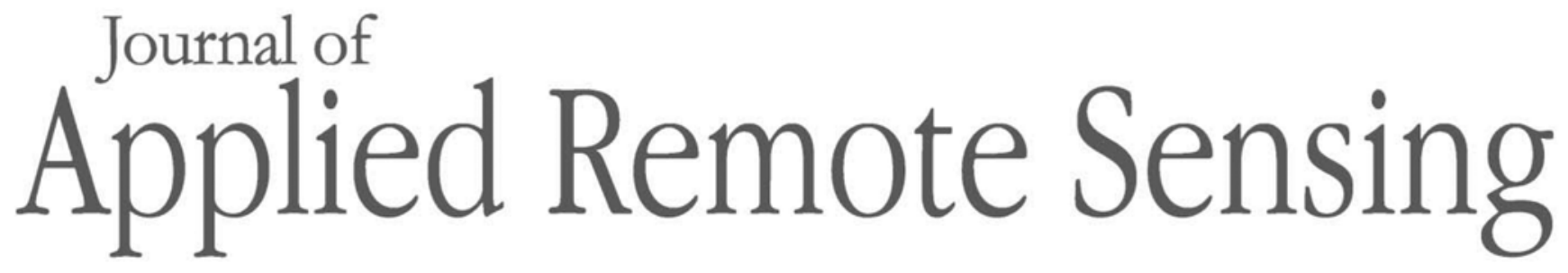

RemoteSensing.SPIEDigitalLibrary.org

\title{
Estimation of secondary forest parameters by integrating image and point cloud-based metrics acquired from unmanned aerial vehicle
}

Ziqian Xu

Weizheng Li

Yuanyuan Li

Xin Shen

Honghua Ruan 


\title{
Estimation of secondary forest parameters by integrating image and point cloud-based metrics acquired from unmanned aerial vehicle
}

\author{
Ziqian Xu, ${ }^{\text {a,b }}$ Weizheng Li, ${ }^{\mathrm{b}}$ Yuanyuan Li, ${ }^{\mathrm{a}, \mathrm{b}}$ Xin Shen, ${ }^{\mathrm{b}}$ and \\ Honghua Ruan ${ }^{\mathrm{a}, \mathrm{b}, *}$ \\ ${ }^{a}$ Nanjing Forestry University, College of Biology and the Environment, \\ Nanjing, Jiangsu, China \\ ${ }^{b}$ Nanjing Forestry University, Co-Innovation Center for Sustainable Forestry in \\ Southern China, Nanjing, Jiangsu, China
}

\begin{abstract}
To better assess spatial variabilities in subtropical secondary forest biomass, the goal of the present study was to employ small-footprint, discrete-return light detection and ranging and unmanned aerial vehicles, integrated with structure from motion (UAV-SFM) data, to accurately estimate the stand characteristic parameters of subtropical forests. The Lorey's height, density, basal area, volume, and aboveground biomass (AGB) of 30 plots $(\varnothing 10 \mathrm{~m})$ were modeled using an array of SFM point cloud metrics and image color metrics (forestry vegetation coverage and color index set). Further, the individual models developed using stepwise multiregression analysis and a branch-and-bound algorithm were employed to examine the best models. Overall, the results indicated that the coefficient of determination $\left(R^{2}\right)$ for Lorey's height $\left(R^{2}=0.58\right.$ to 0.95$)$, volume $\left(R^{2}=0.29\right.$ to 0.71$)$, and $\mathrm{AGB}\left(R^{2}=0.27\right.$ to 0.64$)$ were significantly enhanced compared with the density $\left(R^{2}=0.18\right.$ to 0.48$)$, and basal area $\left(R^{2}=0.24\right.$ to 0.52 ). Utilizing independent stand-level data from ground inventory, our results revealed that, overall, the model fit was significant for most stand characteristic parameters, with relationships close to a 1:1 line, thereby indicating no significant bias [relative root mean square error $($ rRMSE $)=5.32 \%$ to $27.04 \%$ ]. Our image metrics had a statistically significant association with Lorey's height (rRMSE decreased by $0.08 \%$ ), volume (rRMSE decreased by $0.58 \%$ ), and AGB (rRMSE decreased by $3.11 \%$ ), which provided additional explanatory power in the regression analysis. This research demonstrated the potential of UAV-SFM as a technology to accurately assess subtropical forest carbon while providing an improved elucidation of the positive effects of image color metrics. (C) The Authors. Published by SPIE under a Creative Commons Attribution 4.0 Unported License. Distribution or reproduction of this work in whole or in part requires full attribution of the original publication, including its DOI. [DOI: 10.1117/1.JRS.14.022204]
\end{abstract}

Keywords: unmanned aerial vehicle; light detection and ranging; image color; structure from motion; forest stand characteristic parameters.

Paper 190466SS received Jun. 19, 2019; accepted for publication Aug. 20, 2019; published online Sep. 12, 2019.

\section{Introduction}

Forests comprise indispensable components of terrestrial ecosystems while serving as primary global carbon sinks, which play a critical role in offsetting global warming. ${ }^{1,2}$ Researchers have suggested that the carbon emissions from forests generated by deforestation and decomposition account for $17 \%$ of the global total. Therefore, the protection of forest carbon sinks is an essential measure toward dealing with global climate change. ${ }^{3}$ Due to the complexity and dynamic characteristics of forest ecosystems, assessments of multiscale forest carbon storage have typically been highly ambiguous. The estimation of forest carbon reserves typically relies on accurate data of aboveground biomass (AGB), which is generally believed to contain the majority of forest carbon reserves. ${ }^{4-6}$

*Address all correspondence to Honghua Ruan, E-mail: hhruan@njfu.edu.cn 
Initial surveys of forest AGB were conducted through the destructive sampling of trees. A strong correlation was found between the parameters of forest spatial structures [e.g., tree height, diameter at breast height (DBH), etc.] and AGB. ${ }^{7,8}$ The typical biomass of tree species may be more precisely calculated through the establishment of an allometric growth equation. The estimation of biomass by integrating stand characteristic parameters with allometric growth equations has emerged as the primary method for gauging forest inventories, and thus, their carbon storage capacities. ${ }^{9}$

Remote sensing technologies have unique advantages for capturing forest characteristic parameters, which have been widely utilized over the last few decades. ${ }^{10-12}$ With continuous advances in sensors and associated platforms, as well as the enrichment and improvement of various spectral, spatial, and temporal resolutions, forestry researchers can use remote sensing data in combination with ground survey data to obtain accurate profiles of spatial structures of forests.

Numerous studies have revealed that optical remote sensing (particularly hyperspectral remote sensing) may accurately estimate the biomass of forest vegetation, ${ }^{13}$ however, there were limitations in sampling frequency and a saturation of optical data. ${ }^{14-17}$ The emergence of light detection and ranging (LiDAR) greatly improved the accuracy of the estimation of characteristic forest parameter and biomass, as it has the capacity to penetrate the tree canopy and reconstruct vertical forest structures. LiDAR has been verified as suitable for the estimation of parameter and biomass inversion of various forest types and has considerable reliability. ${ }^{18-20}$ However, it has several shortcomings, such as costly data acquisition and lack of spectral information. ${ }^{19,21,22}$

With the rapid development of computer technologies in recent years, unmanned aerial vehicle (UAV) aerial photogrammetry has been rapidly propagated for forestry applications. Due to the high flexibility and low cost of UAV platforms, they have gradually become an important means of data acquisition for forest ecosystem surveys. ${ }^{23,24}$ The application of low-cost UAV-DAP point clouds in forest inventories was introduced by Wallace et al. in $2011 .{ }^{25}$

Currently, several studies have undertaken corresponding experiments with the application of UAV digital aerial photogrammetry (DAP) as relates to the acquisition of stand characteristic parameters and estimation of biomass, which may be approximated by LiDAR. Puliti et al. ${ }^{26}$ acquired high spatial and temporal resolution images from a fixed-wing UAV in southeastern Norway to obtain high-resolution DAP image data. The UAV-DAP metrics were extracted from DAP point clouds, after which the linear models were fitted with metrics for predicting stand characteristic parameters, such as Lorey's height, DBH, etc. Otero et al. ${ }^{27}$ investigated the use of UAV imagery for retrieving structural information on mangrove stands in Malaysia, which suggested that UAV were most useful for the retrieval of canopy height and biomass data from forests that were relatively homogeneous with a single dominant layer.

To date, most of these studies involved small experimental areas (e.g., well-managed, singlelayered, even-aged stands, or woodland forests), and there have been few studies that employed the spectral information contained in UAV images in combination with structural metrics for the estimation of forest biophysical parameters. ${ }^{28,29}$ The types and structures of sample plots were relatively simple and adopted a fixed method for the construction of models, where the specific color data of UAV images were not fully applied. . $^{30,31}$

For this paper, a natural secondary forest in the northern subtropical zone of Zijin Mountain (Niushan) in the eastern suburb of Nanjing, Jiangsu Province, China, was selected as a research zone for the exploration of technical methods toward estimating common stand characteristics through the integration of high-resolution UAV images and LiDAR point cloud data. The purpose of this study was to: (1) elucidate experimental strategies toward the construction of vegetation canopies through LiDAR and DAP three-dimensional (3-D) point clouds, which normalize height data and extract characteristic metrics related to the height and density of canopy point clouds; (2) estimate Lorey's height, density, basal area, volume, and AGB based on an optimal (highest decision coefficient) model; and (3) extract vegetation coverage and color indices from digital UAV images, clarify their participation in modeling, and discuss their impact on modeling accuracy. In sum, we compared the color and noncolor models and developed a color metrics prediction model to obtain a better overall understanding to support subtropical forest management operations. 


\section{Materials and Methods}

\subsection{Study Area}

The study area was a subtropical secondary forest research zone, located in the eastern suburb of Nanjing City, Jiangsu Province, China, which is an extension of Zijin Mountain (Niushan). Specifically, the location is in the northeast portion of Nanjing Forestry University (Fig. 1), with the geographic coordinates of $118.80^{\circ} \mathrm{E}$ and $32.07^{\circ} \mathrm{N}$. The total area was $13.90 \mathrm{hm}^{2}$, with a length of $510 \mathrm{~m}$ from north to south, and width of $720 \mathrm{~m}$ from east to west. The terrain belongs to the southern Jiangsu hills, where the northwest slope is steep, the southeast is relatively flat, and the elevation ranges from 16 to $40 \mathrm{~m}$.
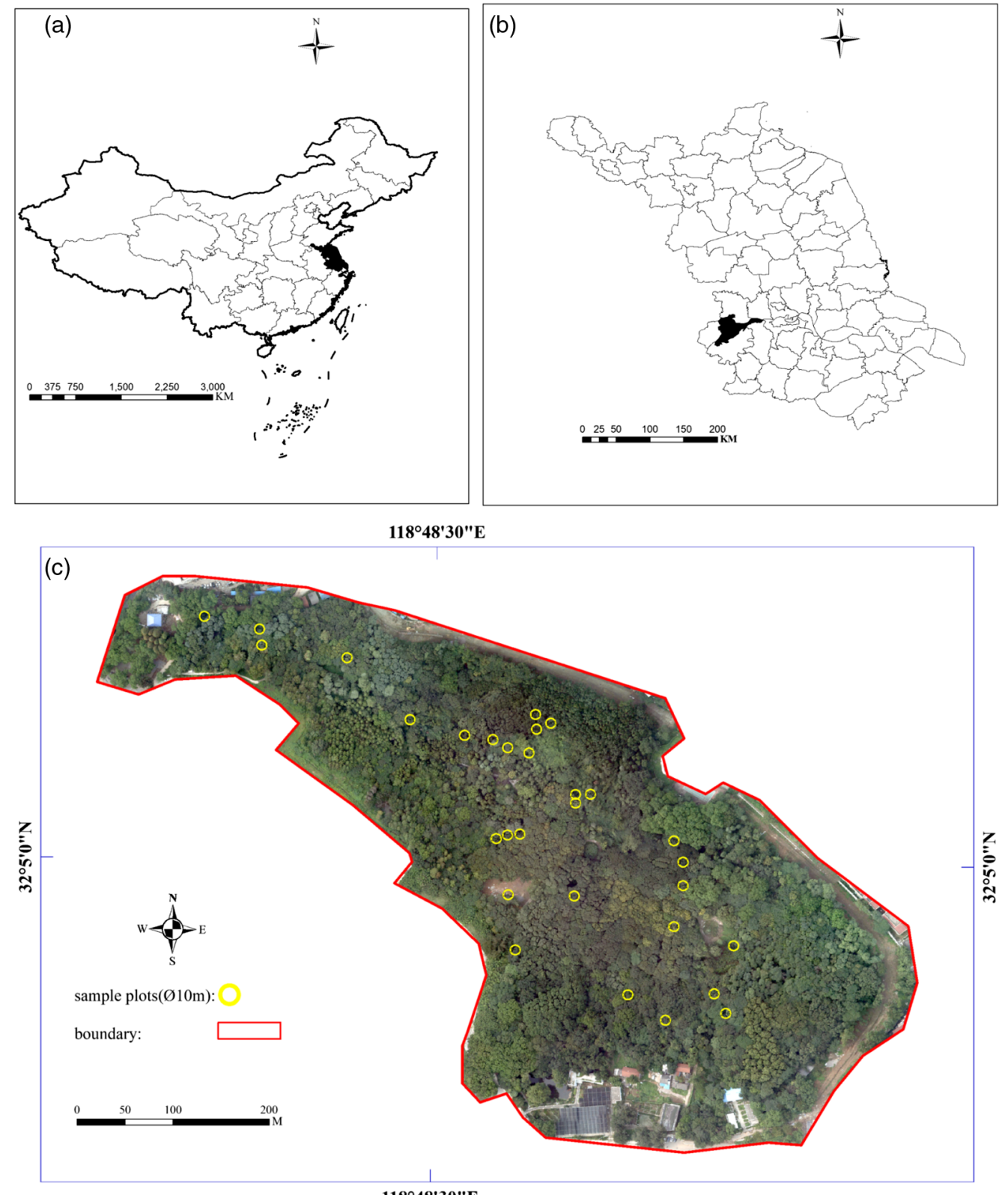

$118^{\circ} 48^{\prime} 30^{\prime \prime} \mathrm{E}$

Fig. 1 Study area and sample plots. (a) Jiangsu Province in China, (b) Nanjing Forestry University in Jiangsu, and (c) the study area and sample plot distribution (c). Coordinate system: GCS_Xian_1980. 
The study area was rich in vegetation with a coverage of $>80 \%$. The main forest types were deciduous broadleaved forest with evergreen components. The primary tree species were beech (Zelkova errata), paper mulberry (Broussonetia papyrifera), privet (Ligustrum ucidum), wingceltis (Pteroceltis atarinowii), with a small percentage of China savin (Juniperus chinensis), and cryptomeria (Cryptomeria japonica var. sinensis).

\subsection{Methods}

For this study, a 3-D forest canopy height model (CHM) was developed based on a sequence of high-precision digital elevation models (DEMs), acquired from LiDAR point clouds, combined with structure from motion (SFM) point clouds, extracted from UAV digital image pairs. Characteristic and color metrics [color index set and forestry vegetation coverage (FVC)] and forest characteristic metrics prediction models were extracted, which were related to height and canopy point cloud density. The specific steps were as follows (Fig. 2): first, UAV images were generated through image registration and feature matching to form image sequences, whereas digital 3-D point clouds and orthophoto images were simultaneously generated via a SFM algorithm and image mosaic. Second, LiDAR point cloud data were employed to produce ground points through a spatial filtering algorithm, combined with orthophoto images and a spatial interpolation algorithm, to create a DEM of the research area. Third, following denoised processing, the aligned SFM point clouds were normalized by the LiDAR-generated DEM to obtain aboveground heights and normalized SFM point clouds. Finally, the point cloud metrics in CHM were extracted, and the color metrics (vegetation coverage and color index) were extracted from the orthophoto images at the sample level. Correlation analysis was then performed using field data, and the prediction model of each characteristic variable was established through the multiple linear regression method. Finally, by comparing the estimation accuracy of the models, with and without color metrics, the influences of the UAV image color data on the model were explored.

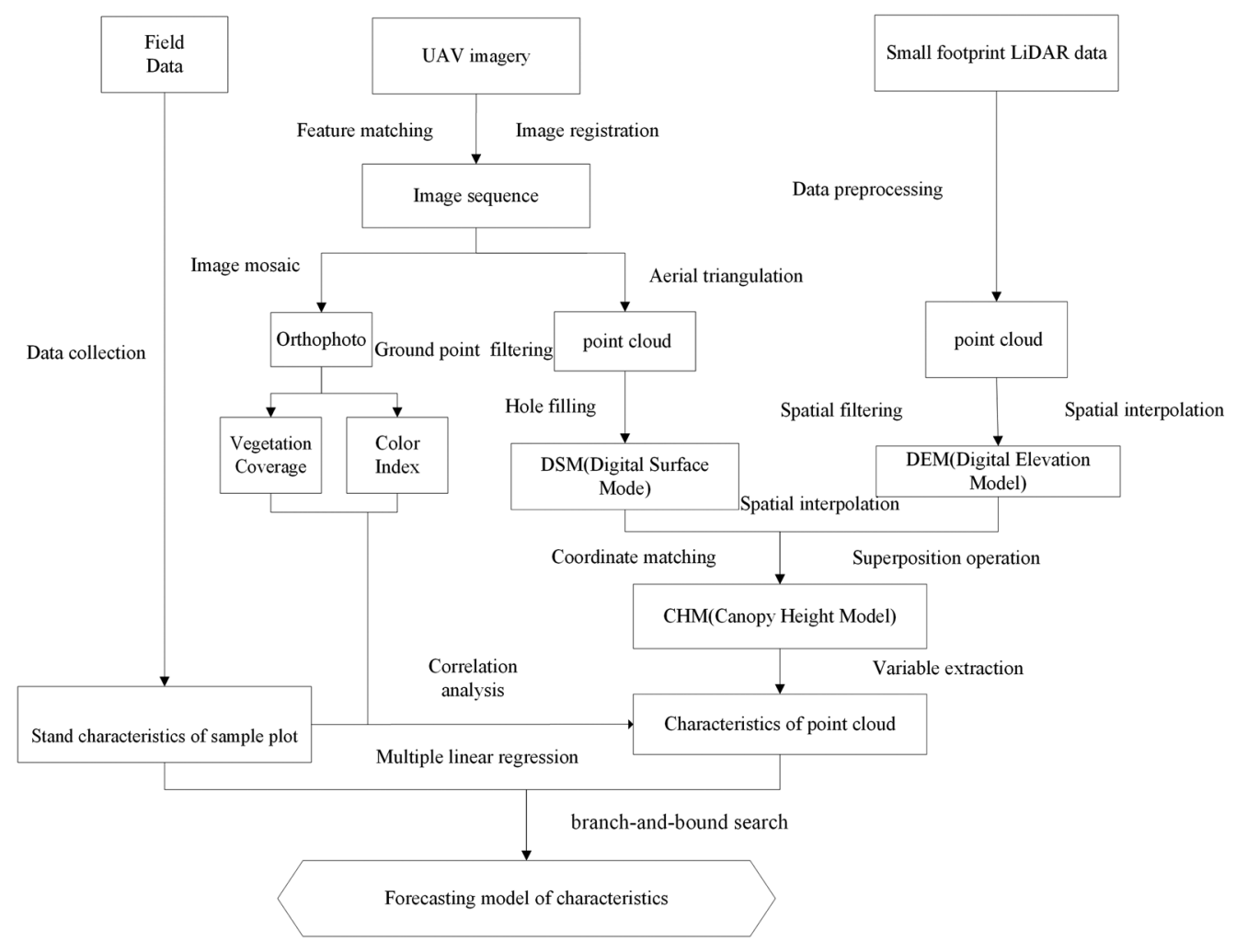

Fig. 2 Flowchart showing the technical process. 


\subsection{Field Data}

A total of thirty $78.54 \mathrm{~m}^{2}(\varnothing 10 \mathrm{~m})$ plots were randomly established within the study site, which encompassed a range of species compositions, ages, and site indices (Fig. 1). The plot data were collected in August 2013, and the center coordinates of the sample plots were located using Trimble GeoExplorer 3000 handheld GPS units augmented with satellite-based augmentation systems to enhance the accuracy and integrity of the GPS data, which resulted in submeter accuracy. $^{32}$

For each plot, all live trees with a DBH $>5 \mathrm{~cm}$, species, diameter, height, height to crown base, and crown width in both cardinal directions were measured. Small trees $(\mathrm{DBH}<5 \mathrm{~cm})$ and dead wood were also tallied for the total stem number. However, they were not used in the biomass calculations. The DBH was measured for all trees with a diameter tape, whereas all of the tree heights were measured using a Vertex IV hypsometer. Several plot-level forest metrics were calculated based on individual tree data, including basal area (cross-sectional area of the trunk at $1.3 \mathrm{~m}$ aboveground) and Lorey's height (i.e., the basal area weighted height).

The DBH and height measurements were loaded into the Chinese Forest Carbon Measurement and Monitoring database program for the derivation of (by province) speciesspecific information and allometric equation data ${ }^{33}$ in Table 1 . Allometric equations developed with tree inventory data from local or nearby provinces were selected for this research, and the tree-based biomass estimates were summed per plot to determine the plot-level biomass components. According to the equations, interannual variations of stand characteristic parameters were minimal, ${ }^{34-36}$ and calculations were made directly from sample plot data. A summary of the derived ground stand characteristic parameters is shown in Table 2.

\subsection{LiDAR Data and Processing}

The LiDAR data acquisition took place on August 18, 2012, operating at a flight altitude of $800 \mathrm{~m}$ aboveground level and a flight speed of $2.8 \mathrm{~m} / \mathrm{s}$. The sensor used in this study was an Optech Airborne Laser Terrain Mapper (ALTM) 3100 laser sensor (Optech Canada, Toronto, Canada). The pulse transmitting frequency of the ALTM3100 sensor was $50 \mathrm{kHz}$, and the scanning frequency was $40 \mathrm{~Hz}$ (scanning angle $+15 \mathrm{deg}$.). The laser spot radius was $30 \mathrm{~cm}$, and the average point distance was $0.83 \mathrm{~m}$. A $1-\mathrm{m}$ DEM was created in two steps from the point cloud data.

Table 1 One-variable volume formula of Jiangsu Province.

\begin{tabular}{|c|c|c|}
\hline Tree species & Formula & Remark \\
\hline Cunninghamia lanceolata & $V=A \times D^{B} \times(E \times D+G \times \lg D)^{C}$ & $\begin{array}{c}A=0.000058777042, B=1.9699831 \\
C=0.89646157, E=-2.2426 \\
F=0.2021, G=6.6922\end{array}$ \\
\hline $\begin{array}{l}\text { Metasequoia } \\
\text { glyptostroboides }\end{array}$ & $V=A \times D^{B} \times\left[\left(E+F \times e^{(G \times D)}\right)^{H}\right]^{C}$ & $\begin{array}{c}A=0.000058777042, B=1.9699831 \\
C=0.89646157, E=1.000438 \\
F=-0.00024755, G=-0.07897864 \\
H=7101.252\end{array}$ \\
\hline Platycladus orientalis & & $\begin{array}{c}A=0.000091972184, B=1.8639778 \\
C=0.83156779, E=1.000084 \\
F=-0.0000671125, G=-0.1223273 \\
H=29416.66\end{array}$ \\
\hline Populus and Quercus & & $\begin{array}{c}A=0.000050479055, B=1.9085054 \\
C=0.99076507, E=0.9236004 \\
F=0.0502109, G=-0.09686479 \\
H=-37.80742\end{array}$ \\
\hline $\begin{array}{l}\text { Salvadoraceae, } \\
\text { Erythrinavariegata Linn., } \\
\text { Salix, other }\end{array}$ & & $\begin{array}{c}A=0.000050479055, B=1.9085054 \\
C=0.99076507, E=6.569053 \\
F=-4.565682, G=-0.03200782 \\
H=1.697762\end{array}$ \\
\hline
\end{tabular}

Note: $\mathrm{D}, \mathrm{DBH}$. 
Xu et al.: Estimation of secondary forest parameters by integrating image...

Table 2 Summary of plot-level forest parameters.

\begin{tabular}{lcc}
\hline \hline & \multicolumn{2}{c}{ Statistics $(n=30)$} \\
\cline { 2 - 3 } Plot-level forest parameters (unit) & Range of variation & Average \\
\hline Lorey's height $(\mathrm{m})$ & $7-31.65$ & 22.99 \\
Density $(\mathrm{n} / \mathrm{ha})$ & $1146-3950$ & 2560 \\
Basal area $\left(\mathrm{m}^{2} / \mathrm{ha}\right)$ & $2.48-13.23$ & 6.41 \\
Volume $\left(\mathrm{m}^{3} / \mathrm{ha}\right)$ & $66.36-488.97$ & 274.36 \\
AGB $(\mathrm{t} / \mathrm{ha})$ & $34.25-156.89$ & 88.16 \\
\hline \hline
\end{tabular}

First, the data were filtered to remove the aboveground returns, and second, the DEM was created by calculating the average elevation from the remaining (ground) LiDAR returns within a particular cell (cells that contained no points were filled via interpolation from neighboring cells). Normalized point clouds were subsequently extracted for each plot using the coordinates of the lower left and upper right corners.

\subsection{UAV Data}

UAV red-green-blue (RGB) imagery was collected for both sources on August 20, 2012. The images were acquired using a Trimble UX5 (Trimble Navigation, Sunnyvale, California) small fixed-wing UAV platform, which was equipped with a Canon EOS 5D Mark II RGB camera (Canon, Japan) and APS-C CMOS image sensor payload. In the flight planning phase, we employed a flight simulation software package to obtain an appropriate flight plan. Flight altitude as well as longitudinal and lateral overlap of the images were set to $800 \mathrm{~m}, 70 \%$, and $30 \%$, respectively. However, the actual flight altitude differed slightly from the defined flight altitude due to wind. All images were collected at noon, and simultaneously, 18 ground control points were set within the study area and located by the trimble real-time kinematic. Image preprocessing was performed using the SFM algorithm, as implemented in Pix4D (Version 4.2.26) (SenseFly, Switzerland) to generate the point clouds. The SFM point clouds were normalized using the LiDAR-DEM. The total number of LiDAR point clouds in the study area was 22,730 (equivalent to $\sim 0.17$ points $/ \mathrm{m}^{2}$ ), and the number of SFM point clouds was 772,916 $\left(\sim 5.56\right.$ points $\left./ \mathrm{m}^{2}\right)$. The spatial operation premise was satisfied by the uniform interpolation of a $1 \times 1 \mathrm{~m}^{2}$ raster.

\subsection{Point Cloud Metrics}

Modeling data metrics were extracted from the normalized point clouds. Referring to the results of the LiDAR point cloud research, ${ }^{37,38}$ this study selected high-level quantile groups (p10, p25, p30, p60, p75, and p90), namely, the quantile of the vertical distribution of forest canopy heights $(10,25,30,40,60,75,85$, and 90 quantile) and corresponding density variable groups (d10, $\mathrm{d} 25, \mathrm{~d} 30, \mathrm{~d} 40, \mathrm{~d} 60, \mathrm{~d} 75, \mathrm{~d} 85$, and d90). The standard deviation ratio of the point cloud heights to mean values was derived from the percentage of point clouds above the percentile height level in all point clouds; the average normalized height value (average height, $h_{\text {mean }}$ ); the maximum value of normalized height (maximum height, $h_{\max }$ ); the minimum value of normalized height (minimum height, $h_{\mathrm{min}}$ ); and height variation (coefficient, $h_{\mathrm{cv}}$ ). Simultaneously, to avoid any disturbance from the lower vegetation on canopy analysis, point clouds under $2 \mathrm{~m}$ in height were removed.

\subsection{Image Color Metrics}

\subsubsection{Forest vegetation coverage}

The FVC was obtained from the orthophoto image of the study area, where the process was as follows. Initially, a UAV image sequence was generated as a true color orthophoto image using 


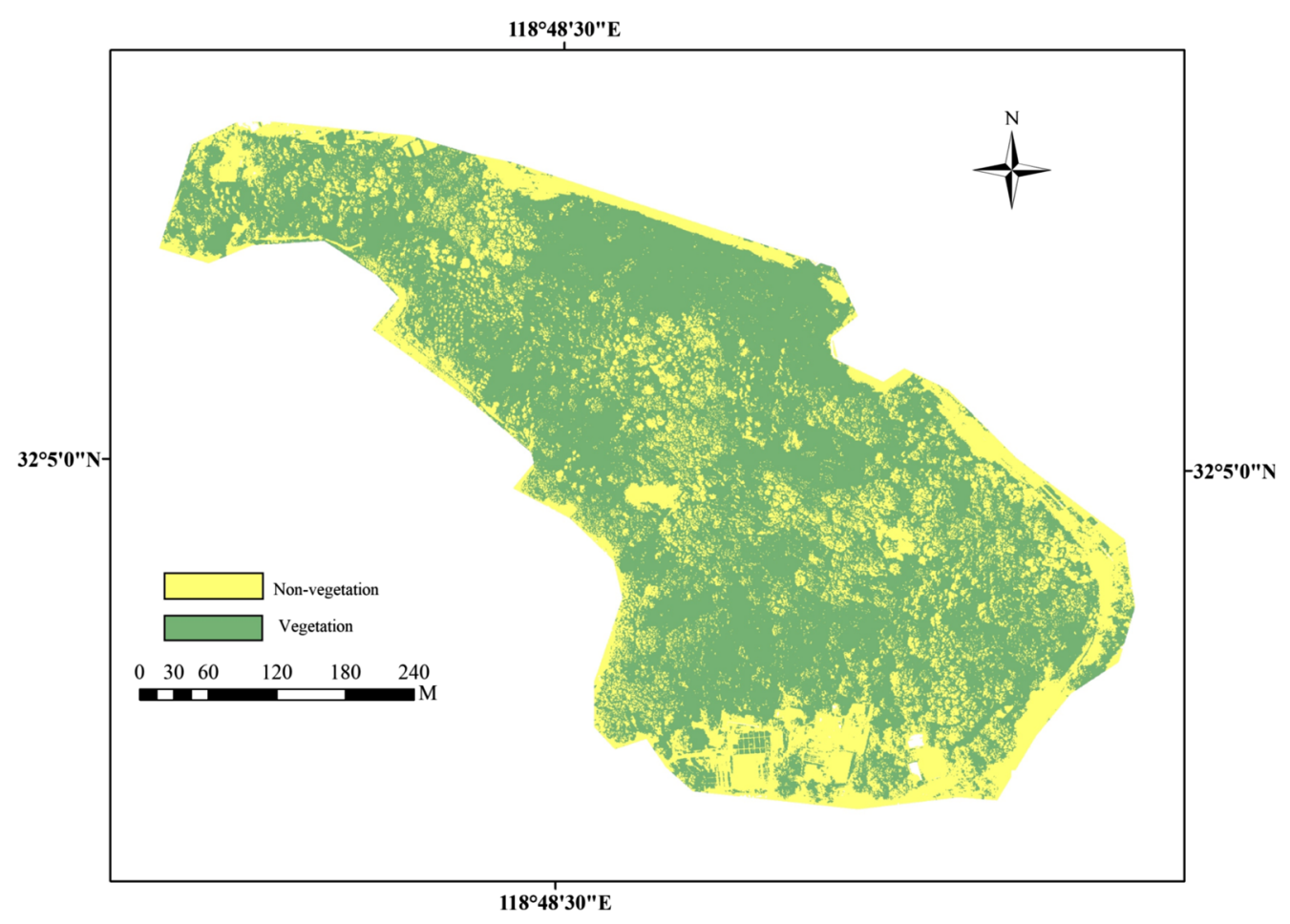

Fig. 3 Classification results of vegetation and nonvegetation in the study area.

image mosaic technology. Second, the visual interpretation was obtained through the sampling of typical features in the study area (combined with field investigations) and classification of vegetation and nonvegetation by supervised classification. ENVI5.1 software was employed for classification, where the overall accuracy of the classification results was $92 \%$, and the Kappa coefficient was 0.89, with results shown in Fig. 3. Finally, FVC was utilized to calculate the ratio of the number of vegetation pixels to the total number of pixels in each plot. ${ }^{39}$

\subsubsection{Color index set}

Since the color value was a critical feature of the SFM point clouds, the color features of the SFM point clouds were stored as RGB data for each point. In contrast to traditional satellite and aerial remote sensing, the vegetation index was calculated in the near-infrared, where the red bands represented vegetation. Due to the lack of near-infrared bands, the UAV images could only extract vegetation information by mining the visible light color data. Currently, the color index set based on traditional digital camera images included primarily the excess green (EXG) index, excess green minus excess red (ExG-ExR) index, and normalized green-red difference index (NGRDI). ${ }^{40,41}$

\section{NGRDI}

The formula for calculating the NGRDI is as follows [Eq. (1)]:

$$
\mathrm{NGRDI}=(G-R) /(G+R) .
$$

In the above formula, $G$ and $R$ are the DN values of the green and red bands. The NGRDI eliminated the influence of different irradiances on the vegetation spectral characteristics by normalizing the differences between the green and red bands. The NGRDI values ranged from -1 to +1 .

2. EXG index, EXR index, and ExG-ExR index

By subtracting the EXG index from the EXR index, the ExG-ExR index could be obtained. The calculation formulas are as follows [Eqs. (2)-(10)]: 
Xu et al.: Estimation of secondary forest parameters by integrating image...

$$
\mathrm{ExG}-\mathrm{ExR}=3 g-2.4 r-b .
$$

In the above formula, ExG and ExR are excess-green and excess-red indices, respectively. The calculation formulae are as follows:

$$
\begin{gathered}
\mathrm{ExG}=2 g-r-b, \\
\mathrm{ExR}=1.4 r-g .
\end{gathered}
$$

In the above formulae, $g, r$, and $b$ are green, red, and blue band coordinates, respectively, and the three are one, in which:

$$
\begin{aligned}
& R=R * /(R *+G *+B *), \\
& G=G * /(R *+G *+B *), \\
& B=B * /(R *+G *+B *) .
\end{aligned}
$$

In the above formulae, $R *, G *, B *$ are normalized $\mathrm{DN}$ values in red, green, and blue bands, i.e.,

$$
\begin{aligned}
& R *=R / R_{\max }, \\
& G *=G / G_{\max }, \\
& B *=B / B_{\max } .
\end{aligned}
$$

In the above formulae, $R_{\max }, G_{\max }$, and $B_{\max }$ are the maximum values of the threecolor components, typically 255 in 24-bit storage and 65,535 in 32-bit storage. By subtracting the two, the vegetation and background can be well distinguished. Referring to previous research results, the EXG index, ExG-ExR index, NGRDI, and green leaf index (GLI) were selected as modelling factors (Fig. 4), and GLI was the green-band coordinate value $G$.

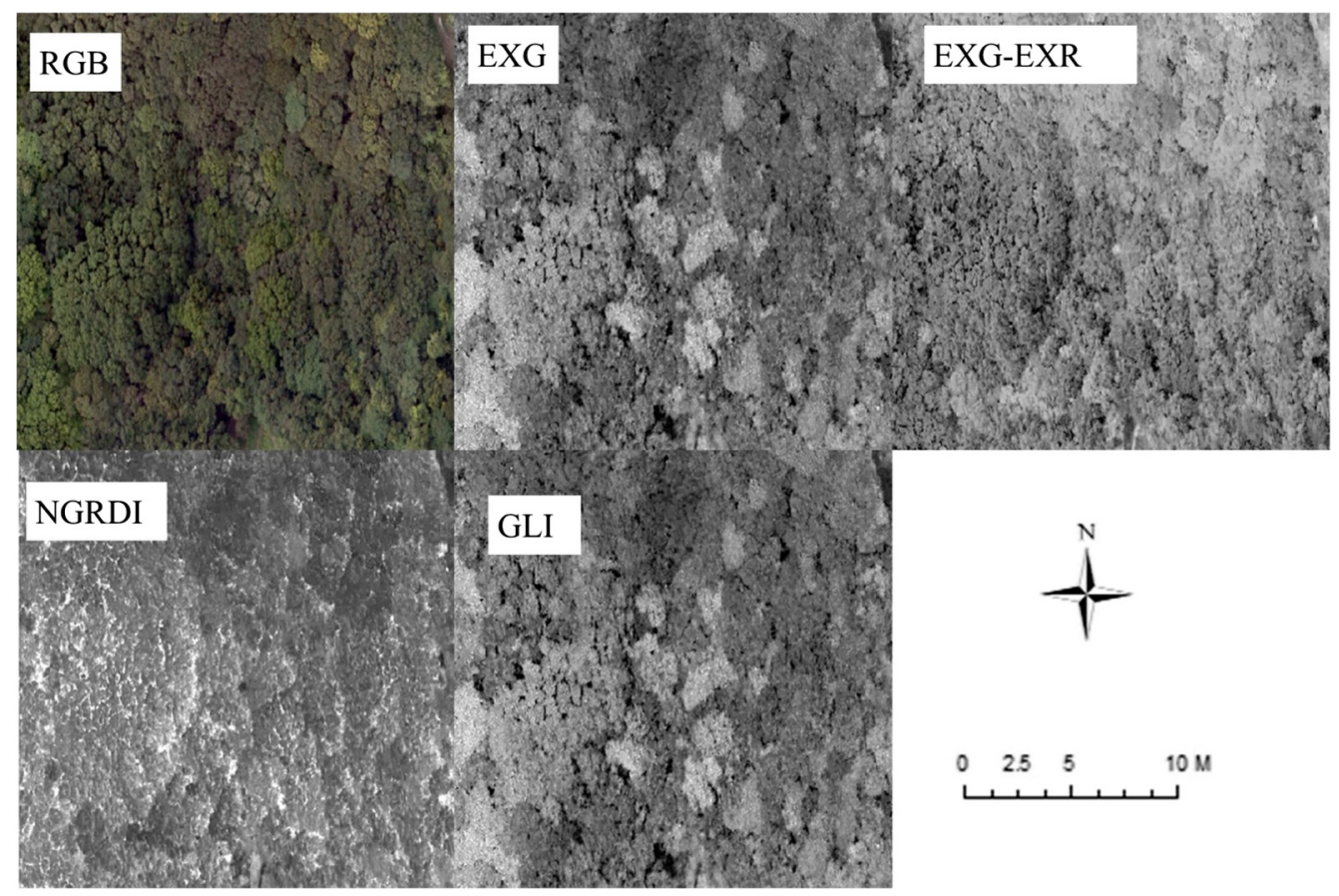

Fig. 4 Images of the sample area in the original RGB format prior to manipulations, EXG index values, EXG-EXR index, and GLI values. 
Table 3 Metrics for modeling.

\begin{tabular}{|c|c|c|}
\hline Independent variable & & Dependent variable \\
\hline Height percentile & $(\mathrm{p} 10, \mathrm{p} 25, \mathrm{p} 30, \mathrm{p} 40, \mathrm{p} 60, \mathrm{p} 75, \mathrm{p} 85, \mathrm{p} 90)$ & $\begin{array}{l}\text { Lorey's height }(\mathrm{m}) \\
\text { density }(\mathrm{n} / \mathrm{ha})\end{array}$ \\
\hline Density metrics & $(\mathrm{d} 10, \mathrm{~d} 25, \mathrm{~d} 30, \mathrm{~d} 40, \mathrm{~d} 60, \mathrm{~d} 75, \mathrm{~d} 85, \mathrm{~d} 90)$ & $\begin{array}{c}\text { Basal area }\left(\mathrm{m}^{2} / \mathrm{ha}\right) \\
\text { Volume }\left(\mathrm{m}^{3} / \mathrm{ha}\right)\end{array}$ \\
\hline Height variation metrics & $h_{\text {mean }}, h_{\max }, h_{\min }, h_{\mathrm{cv}}$ & AGB (t/ha) \\
\hline Image color metrics & FVC, EXG, ExG-ExR, NGRDI, GLI & \\
\hline
\end{tabular}

\subsection{Metrics Selection and Modeling Analysis}

For this study, a total of four categories and 25 metrics were involved in the development of the model (Table 3). A multivariate linear regression technique was employed for the model, whereas a logarithmic variation was used to eliminate the dimension effect of all metrics. To reduce the independent metrics dimension and collinearity between independent metrics, a subset of simple linear regression models was established for each independent and dependent variable, after which a branch-and-bound search was conducted using the "leaps" package in $\mathrm{R}$ (v3.5) to identify the best regression model. ${ }^{42}$

The number of independent metrics was limited to 5 via Bayesian information criterion, and colinearity detection was carried out by the variance expansion factor $<5$ to eliminate the factors associated with excessive collinearity. To investigate the influence of color metrics on modeling, the subset of models with color metrics, and those without color metrics with the highest fitting degree of the same dependent variable were selected as the optimized models, respectively.

A cross-validation method was employed to validate the model, and the selected indicators were as follows:

1. Determinant coefficient $\left(R^{2}\right)$ : The greater the $R^{2}$ value, the stronger the correlation between dependent and independent variables, which was the test of goodness of fit for the regression straight line Eq. (11):

$$
R^{2}=1-\sum_{i-1}^{n}\left(x_{i}-\hat{x}_{i}\right)^{2} / \sum_{i-1}^{n}\left(x_{i}-\bar{x}_{i}\right)^{2} \text {. }
$$

2. RMSE: Expresses the square root of the deviation between the observed and true values, and the ratio of the observed number $n$ in Eq. (12):

$$
\operatorname{RMSE}=\sqrt{\frac{1}{n} \sum_{i-1}^{n}\left(x_{i}-\hat{x}_{i}\right)^{2}} .
$$

3. rRMSE: Defined as the ratio of RMSE to the arithmetic average of the measured results in Eq. (13). The smaller the rRMSE, the better the prediction effect of the model. This index had nothing to do with the magnitude of the evaluation quantity itself and could better reflect the difference of the model:

$$
\mathrm{rRMSE}=\frac{\mathrm{RMSE}}{\bar{x}_{i}} \times 100 \% .
$$

\section{Results}

\subsection{Correlation Analysis of Point Cloud Metrics}

Due to the high complexity of forest ecosystems, the relationships between point cloud characteristic metrics and field data were very complex and highly uncertain. The selection of metrics 


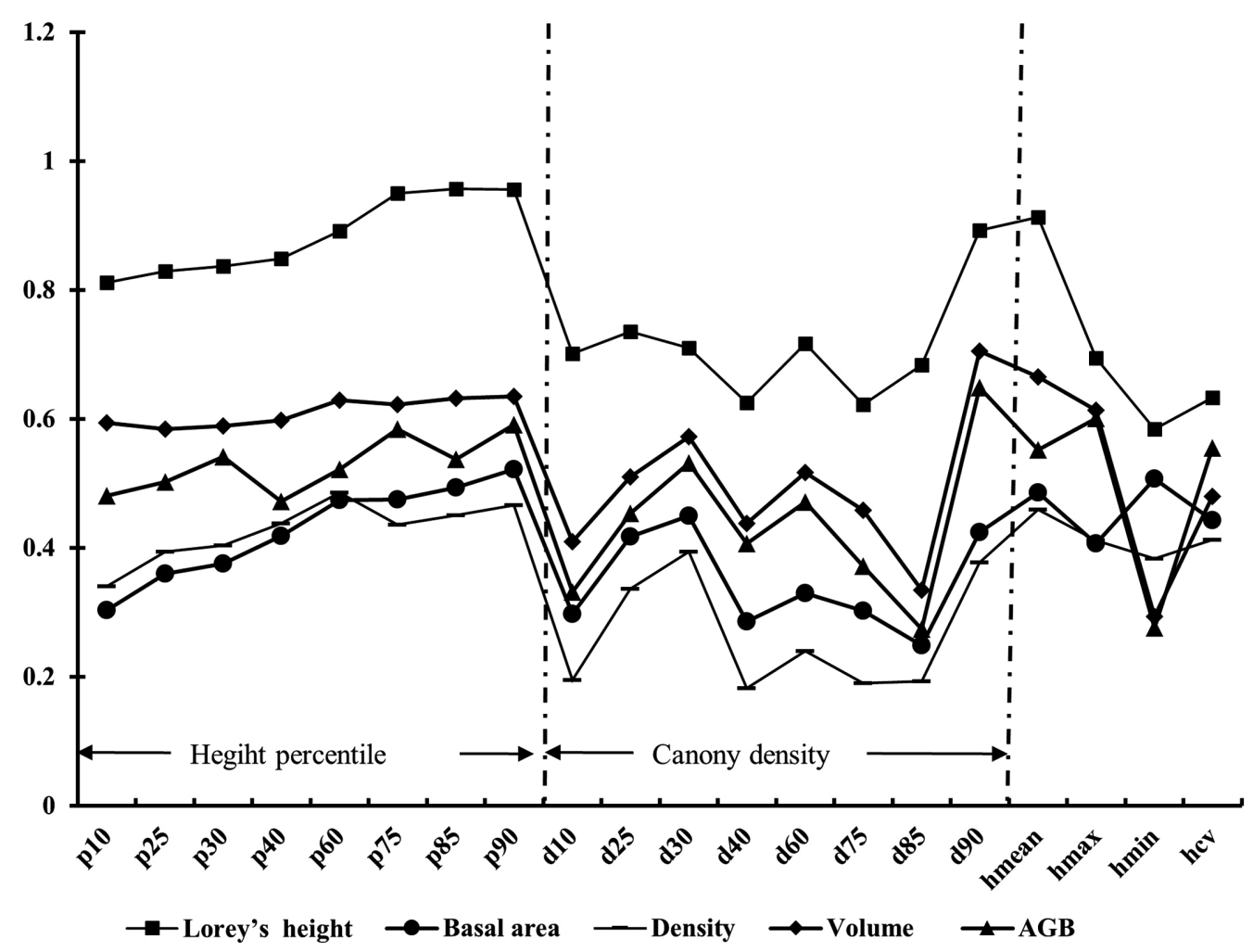

Fig. 5 Coefficients between point-cloud metrics and stand characteristics.

has the requirement of being optimized by a statistical test, which may be found through correlation analysis (Fig. 5). The correlation between the characteristic metrics extracted from normalized point clouds and Lorey's height was the highest $\left(R^{2}=0.58\right.$ to 0.95$)$, followed by the volume $\left(R^{2}=0.29\right.$ to 0.71$)$, AGB $\left(R^{2}=0.27\right.$ to 0.64$)$. The basal area $\left(R^{2}=0.24\right.$ to 0.52$)$ and density $\left(R^{2}=0.18\right.$ to 0.48$)$ were the lowest.

Between the jointly extracted metrics, the correlations between the mean height $\left(h_{\text {mean }}\right)$, high coefficient of variation $\left(h_{\mathrm{cv}}\right)$, high-rank height quantile (p75 to p90), middle-high-rank cloud density (d60, d90), and stand characteristics were also higher. In contrast, the correlations between the maximum height $\left(h_{\max }\right)$, middle-low-rank height quantile, point cloud density, and stand characteristics were lower.

\subsection{Estimation Model Construction and Accuracy Analysis}

The estimation model was constructed through multivariate regression analysis, where the model calculating metrics were in good agreement with the measured metrics $\left(R^{2}=0.29\right.$ to 0.86$)$ (Table 4). The regression equation parameters of each stand were $<5$ in the model parameters. Independent metrics, including height percentile, point cloud density, and mean height, were highly correlated with the corresponding stand characteristics. Lorey's height had the best fit with the stand characteristic metrics, followed by volume, basal area, AGB, and density. The fitting results were essentially consistent with the correlation analysis results.

From the perspective of rRMSE, the order of accuracy from high to low was Lorey's height (5.32\%), volume (6.35\%), AGB (10.41\%), basal area (16.38\%), and density (27.04\%). According to the selected factors of each model, the upper height quantile (P90, P60) was selected most frequently (Lorey's height, density, volume, and AGB), followed by the height mean $\left(h_{\text {mean }}\right)$, which was selected by Lorey's height, volume, and the AGB model. Density metrics and the lower-rank height quantile participated in Lorey's height and basal area modeling.

Following the model regression search, color variables were selected through factor models other than density and basal area, which resulted in the prediction accuracy of the 
Xu et al.: Estimation of secondary forest parameters by integrating image...

Table 4 Estimation models and accuracy assessments.

\begin{tabular}{|c|c|c|c|c|}
\hline Stand characteristics & Combined extraction estimation models & $R^{2}$ & RMSE & rRMSE (\%) \\
\hline \multirow[t]{2}{*}{ Lorey's height $(\mathrm{m})$} & $\ln H=0.23+0.579 \bullet \operatorname{In} h_{\text {mean }}+0.346 \bullet \operatorname{Inp} 90$ & 0.86 & 0.13 & 6.47 \\
\hline & $\begin{aligned} \text { In } H= & -0.79+1.34 \bullet \operatorname{In} h_{\text {mean }} \\
& +0.369 \bullet \operatorname{Ind} 85+4.15 * \ln \mathrm{GIL}\end{aligned}$ & 0.94 & 0.08 & 6.39 \\
\hline Density (n/ ha) & $\ln N=6.39+6.027 \bullet \ln \mathrm{p}_{60}-5.84 \bullet \ln \mathrm{p}_{30}$ & 0.29 & 0.69 & 27.04 \\
\hline Basal area $\left(m^{2} / h a\right)$ & $\ln G=2.752 \bullet \ln p_{60}-1.841 \bullet \ln p_{10}-1.126$ & 0.53 & 0.28 & 16.38 \\
\hline \multirow[t]{2}{*}{ Volume $\left(\mathrm{m}^{3} / \mathrm{ha}\right)$} & $\ln V=2.499+1.429 \bullet \ln p_{90}+0.7 \bullet \ln d_{90}$ & 0.59 & 0.40 & 6.93 \\
\hline & $\begin{aligned} \ln V= & 4.365+0.759 \bullet \ln p_{90} \\
& -7.0223 \bullet \ln h_{\text {mean }}+3.23 \bullet \operatorname{In} \mathrm{FVC}\end{aligned}$ & 0.62 & 0.33 & 6.35 \\
\hline \multirow[t]{2}{*}{ AGB (t/ha) } & $\ln A=15.27+7.893 \bullet \operatorname{lnp} 90-4.68 \bullet \operatorname{Inp} 30$ & 0.55 & 0.35 & 13.25 \\
\hline & $\begin{aligned} \operatorname{In} A= & 23.65-4.986 \bullet \operatorname{In~FVC~} \\
& +3.35 \bullet h_{\text {mean }}-5.233 \bullet \operatorname{In} \text { EXG }\end{aligned}$ & 0.60 & 0.31 & 10.41 \\
\hline
\end{tabular}

Note: $H$, Lorey's height; $N$, density; $G$, basal area; $V$, volume; $A$, AGB.

model being improved. The FVC was selected by the volume and AGB model, the GLI was selected by Lorey's height model, and the EXG index was selected by the AGB model, which revealed that the green-band data had a certain response to tree height, volume, and biomass.

The cross-validation estimates of stand characteristic models and scatter plots of the sample plots are shown in Fig. 6. It can be seen from the graph that the accuracy of Lorey's height recognition was the highest $\left(R^{2}=0.93\right)$, and the fitting equation was basically consistent with a 1:1 validation line. Remarkably, the correlation was quite significant; the accuracies of the basal area and density were relatively low $\left(R^{2}=0.46\right.$ and $\left.R^{2}=0.32\right)$, whereas the accuracies of the volume and AGB were in the middle $\left(R^{2}=0.60\right.$ and $\left.R^{2}=0.58\right)$.

\subsection{Influence of Color Metrics on Model Development}

Color metrics, such as vegetation coverage and the color index set, were introduced into the models as parameters. The models with color metrics were established by Lorey's height, volume, and AGB. Their $R^{2}$ were $0.94,0.62$, and 0.6 , and rRMSE were $6.39 \%, 6.35 \%$, and $10.41 \%$, respectively. The pure point cloud variable models $\left(R^{2}\right)$ of the three factors were $0.86,0.59$, and 0.55 , whereas the rRMSE was $5.32 \%, 6.93 \%$, and $13.52 \%$, respectively. The results revealed that the vegetation coverage and color index set could improve the accuracy of the model to a certain extent, with AGB (rRMSE decreased by $3.11 \%$ ), followed by storage $0.58) \%)$ and Lorey's height $(0.08 \%)$.

A comparison of the measured and estimated values of Lorey's height, volume, and AGB for all plots can be seen in Fig. 7. The variable color models were more accurate in estimating Lorey's height, and the sample plots with large estimation errors in the colorless component model were corrected. The involvement of color components in the estimation of biomass-related factors with low estimates had an increasing effect. In the assessment of biomass, the volume estimated by the model without color metrics was significantly lower than the measured value in nos. 10 and 19 plots. The estimated value of the model increased significantly following the addition of color metrics, which was closer to the measured value.

Similar to the volume, the noncolor variable model of AGB was significantly lower in plot nos. $2,12,15,24$, and 27 , while the color variable model was improved in varying degrees, thus enhancing the estimation accuracy of the model. Simultaneously, the color metrics also increased the original estimated value of the plot (e.g., plot no. 20, AGB plot nos. 9, 28). Therefore, the effect of improving the accuracy of the model was limited. 


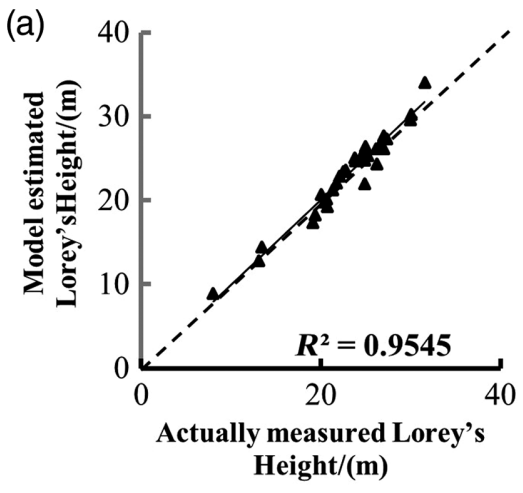

(c)

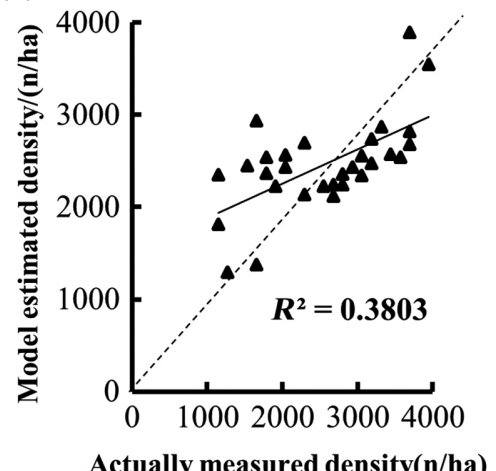

Actually measured density(n/ha)

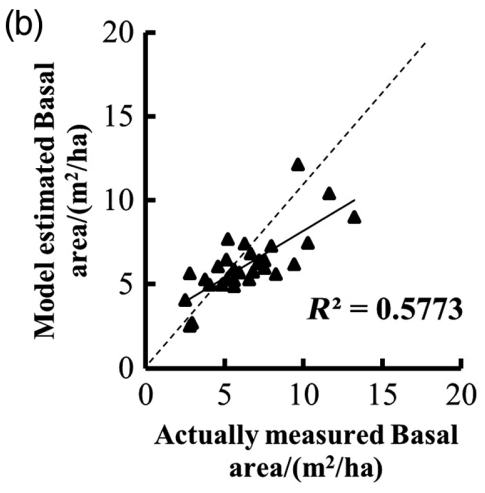

(d)

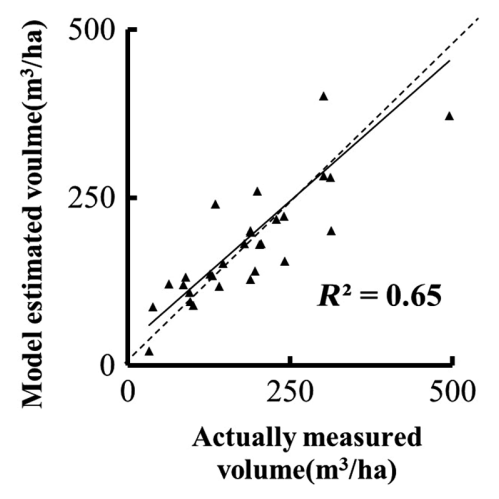

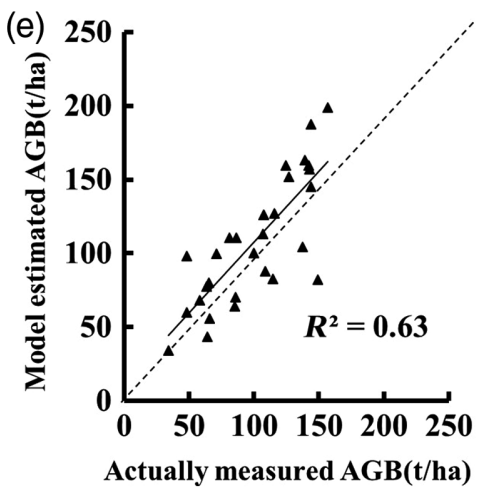

Fig. 6 Comparison of field-measured forest characteristics and model estimation (dotted line is 1:1 validation line). (a) Lorey's height, (b) basal area, (c) density, (d) volume, and (e) AGB.

\section{Discussion}

The independent metrics of the multivariate regression model consisted of three groups of point cloud characteristic metrics: height quantile, point cloud density, and height data. The correlations between the height quantile and forest vertical data were higher, which was similar to some of the LiDAR results. Fu et al. ${ }^{43}$ estimated the structural parameters of Canadian coastal forests using discrete small spot LiDAR point cloud data. The selected model metrics were p50, p30, d60, d90, and so on. The accuracy of the model with canopy return density was higher than those with single height information.

Cao et al. ${ }^{44}$ extracted characteristic LiDAR parameters in the Yushan subtropical forest area of Jiangsu Province and found that $h_{\text {mean }}$, D60, and $h_{\mathrm{cv}}$ were repeatedly selected in different models. As it was difficult to penetrate the canopy, the height percentiles and density components of the UAV images were often higher than LiDAR, and the forest types with more complex canopy results were more obvious. The model parameters obtained in this study also included low-level and maximum factors, such as P10 and $h_{\min }$. The UAV digital images employed in this study captured data for the upper tree crowns and described their height 

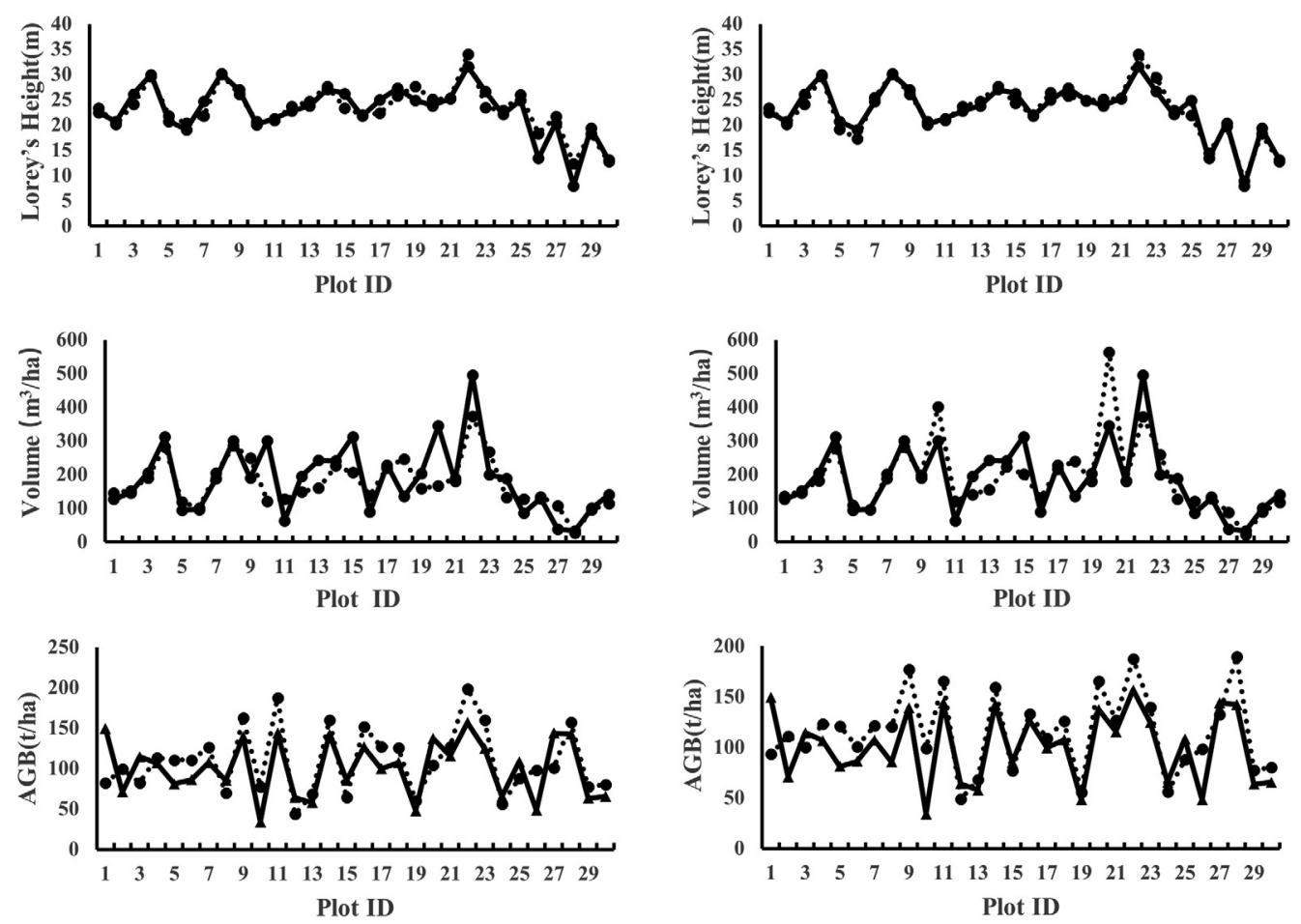

(a) non-color variables model

$\begin{array}{ll}\rightarrow & \text { Filed value } \\ \ldots & \text { predicted value }\end{array}$

(b) color variables model

Fig. 7 Lorey's height, volume, and AGB predicted data by (a) noncolor metrics participation model and (b) color metrics participation model.

fluctuations. However, they could not obtain data related to the lower layers of the tree crowns, which were covered. The low-level height metrics in the SFM data were often equivalent to the middle-level LiDAR data, and the $h_{\text {min }}$ of the SFM was equivalent to the middle-level of LiDAR point clouds of the same density, which was also consistent with previous research results.

Through previous research, the fitting effect and accuracy of the multiregression prediction model of the stand characteristics developed in this study were similar to those of other models using LiDAR point clouds only. Næsset et al. ${ }^{37}$ estimated the characteristic parameters of a Norwegian subfrigid forest based on LiDAR. The results revealed that the $R^{2}$ of the estimation models for the characteristic parameters of this forest were average height ( 0.82 to 0.95$)$, basal area $(0.69$ to 0.89$)$, and volume $(0.80$ to 0.93$)$, which were slightly higher than those of the $R^{2}$ models obtained in this study.

As most of the subtropical forests were multilayered, their structures were more complex (the tree heights and densities varied significantly); thus, the accuracy of the data extraction was low, and inconsistency was high. He et al. ${ }^{45}$ used LiDAR point clouds to extract the individual structural parameters of spruce trees in an alpine zone and applied a multiple stepwise regression analysis method to establish the statistical regression relationship between the average tree height, crown width, measured stand average height, crown width, DBH, density, and AGB at the sample plot scale. The results revealed that the average stand height and stand arithmetic were significant.

The estimation equation of the average height and AGB had higher accuracy $\left(R^{2}>0.7\right)$, while the determination coefficient of the estimation equation of the average crown width, average DBH, and density was slightly lower $\left(R^{2}>0.5\right)$, which were similar to the results of this paper. The focus of this paper was a subtropical secondary forest, the structure of which was more complex than that described in the previous literature. However, due to the high density of DAP point clouds and the small study/sample areas, ideal experimental results were obtained. 
The results of the model calculation revealed that the accuracy of the tree height inversion based on vertical forest data was the highest. The accuracy of the basal area and density inversion based on the horizontal data was lower, while the accuracy of the volume inversion based on the horizontal and vertical information was in the middle.

The Lorey's height, volume, and AGB model accuracies were slightly improved by adding color metrics. At present, multiple studies have shown that the accuracy of models may be enhanced to a certain extent by combining conventional point cloud feature metrics with other factors. Lucas et al. ${ }^{46}$ used LiDAR and CASI data to extract forest biomass data. This research revealed that fused data were more accurate for modeling.

Xu et al. ${ }^{47}$ combined Landsat Operational Land Imager (OLI) with LiDAR data to establish a comprehensive model for estimating aboveground forests and belowground biomass. The results showed that the accuracy of the comprehensive model for estimating aboveground forests and underground biomass was higher than that of the OLI and LiDAR models. The correlations between the OLI texture data and height percentile metrics and biomass were strong. Puliti et al. ${ }^{48}$ added green-band spectral information of UAV images to the modeling of forest characteristic metrics. It was found that the spectral data improved the estimation of Lorey's height, dominant tree height, and density; however, it was not significant (RMSE decreased by $0.38 \%$ at most). Jayathunga ${ }^{49}$ employed vegetation coverage with point cloud height quantile and density quantile to estimate volume and biomass carbon storage in Hokkaido, Japan.

The results revealed that vegetation coverage increased the accuracy of the volume and biomass model (RMSE decreased by $1.9 \%$ and $1.55 \%$ ), respectively, and there was a negative correlation between the vegetation coverage, volume, and biomass. This may have been due to the numerous trees in the research area. Shen et al. ${ }^{50}$ used multispectral and RGB imagery to estimate the attributes of forest structures. The models that included only spectral indices had the capacity to predict the features of forest structures with relatively high accuracy $\left(R^{2}=0.56\right.$ to $0.69, \mathrm{rRMSE}=10.88 \%$ to $21.92 \%$ ). Moreover, the models that were fitted using multispectral and RGB-derived metrics had similar accuracies $\left(\Delta R^{2}=0\right.$ to $0.02, \Delta \mathrm{rRMSE}=0.18 \%$ to $0.44 \%)$.

The results of this study were similar to those above, where models for Lorey's height, volume, and AGB improved estimation accuracies due to the addition of vegetation coverage, GLI, and EXG index. The calculation of the color metrics employed involved the green-band of visible light, which reflected the relationship responses between the vegetation growth and green-band to a certain extent.

The differences observed in the rRMSE values between the different stand characteristic parameters could be related to the structural complexity of the parameters. In this study, the density and basal area exhibited the highest rRMSE, which had the highest variability in structural complexity, in contrast to the other parameters. One possible solution to overcome this issue might be to develop stand-specific regression equations or to use the stand type as the random effect variable in robust linear mixed model. For our future research target, we will address this issue in detail, and how to use UAV photogrammetry in subtropical forest management toward the achievement of sustainability goals.

Although the RMSE of Lorey's height, volume, and AGB was reduced following the addition of the color metrics to the regression models, the estimations with and without color metrics were not statistically significant, which might perhaps be ascribed to the complexity of the forest in our study area. Furthermore, it would be important to test alternative methods that can be employed to improve the accuracy of model estimation. For example, (1) extended modeling factors, such as color index, vegetation index, temperature, precipitation, etc., to extract useful information from mass data for assimilation and integration and (2) use of multisource data to participate in modeling, such as hyperspectral, microwave, etc.

\section{Conclusion}

For this study, we developed predictive forest stand characteristic models using UAV-based LiDAR and DAP data, respectively, in secondary forests within a subtropical zone of East China. Correlation analysis revealed that jointly extracted point cloud metrics were highly 
sensitive to vertical forest data (tree height), followed by volume, AGB, and density, where the basal area was the lowest. Due to the lack of penetration of the UAV digital images, they were less sensitive to density, which were primarily reflected via the density data.

The supervised classification of UAV images was conducted to extract the vegetation coverage of each plot, and the corresponding green index, green-red index, NGRDI, and GLI were calculated. The vegetation coverage and color index datasets were employed as color metrics for modeling. The results revealed that following model selection, color metrics participated in the determination of Lorey's height, volume, and AGB. The quantities of three types of factors were modeled, and the accuracy of the model was improved to a certain extent.

Therefore, our results highlight the potential of UAV images for assessing forest resources to support operational forest management activities in subtropical secondary forests, which are characterized by complex horizontal and vertical structures. This study provided a good example of the capacity of UAV to accurately assess forest stand characteristic parameters. Future studies should focus on determining the impacts of seasonal differences on UAV photogrammetric data, when estimating forest structural attributes.

\section{Acknowledgments}

We acknowledge grants from the National Key Research and Development Program of China (Grant No. 2016YFD0600204) and National Natural Science Foundation of China (Grant Nos. 41501487, 41371421, 41471373, and 41201151). This research was also supported by the Priority Academic Program Development of Jiangsu Higher Education Institutions (PAPD).

We thank Dr. Frank Boehm for checking the writing, and the anonymous reviewers, who significantly contributed toward the improvement of this article.

\section{References}

1. J. Fang et al., "Changes in forest biomass carbon storage in China between 1949 and 1998," Science 292(5525), 2320-2322 (2001).

2. H. E. M. Nascimento and W. F. Laurance, "Total aboveground biomass in central Amazonian rainforests: a landscape-scale study," For. Ecol. Manage. 168(1-3), 311-321 (2002).

3. B. Little, "Under an open sky—career tree planters," American Forests Spring (2001).

4. S. Brown and A. E. Lugo, "Biomass of tropical forests: a new estimate based on forest volumes," Science 223(4642), 1290-1293 (1984).

5. Z. Zhang et al., "Prediction of diameter distributions with multimodal models using LiDAR data in subtropical planted forests," Forests 10(2), 125 (2019).

6. K. Liu et al., "Estimating forest structural attributes using UAV-LiDAR data in ginkgo plantations," ISPRS J. Photogramm. Remote Sens. 146, 465-482 (2018).

7. J. Morgenroth and C. Gomez, "Assessment of tree structure using a 3D image analysis technique-a proof of concept," Urban For. Urban Greening 13(1), 198-203 (2014).

8. L. Cao et al., "Estimating tree volume distributions in subtropical forests using airborne LiDAR data," Remote Sens. 11(1), 97 (2019).

9. Z. Zhang et al., "Predicting LiDAR-derived biomass distributions by Weibull models in a subtropical forest," in Int. Workshop Earth Obs. and Remote Sens. Appl. (2018).

10. C. Lin, R. Ma, and J. Xiong, "Can the watershed non-point phosphorus pollution be interpreted by critical soil properties? A new insight of different soil P states," Sci. Total Environ. 628-629, 870-881 (2018).

11. T. Yun et al., "Rubber tree crown segmentation and property retrieval using ground-based mobile LiDAR after natural disturbances," Remote Sens. 11(8), 903 (2019).

12. L. Chen et al., "Detection of sensitive soil properties related to non-point phosphorus pollution by integrated models of SEDD and PLOAD," Ecol. Indic. 60, 483-494 (2016).

13. X. Shen et al., "The assessment of synergetic effects of airborne LiDAR, CCD and hyperspectral data for above-ground biomass estimation," in Fifth Int. Workshop Earth Obs. and Remote Sens. Appl. (EORSA), pp. 1-5 (2018). 
14. K. H. Gibbs et al., "Monitoring and estimating tropical forest carbon stocks: making REDD a reality," Environ. Res. Lett. 2(4), 045023 (2007).

15. L. I. Duncanson, K. O. Niemann, and M. A. Wulder, "Integration of GLAS and Landsat TM data for aboveground biomass estimation," Can. J. Remote Sens. 36(2), 129-141 (2010).

16. J. R. Hall et al., "Modeling forest stand structure attributes using Landsat ETM+ data: application to mapping of aboveground biomass and stand volume," For. Ecol. Manage. 225(1), 378-390 (2006).

17. D. Lu, "Aboveground biomass estimation using Landsat TM data in the Brazilian Amazon," Int. J. Remote Sens. 26(12), 2509-2525 (2005).

18. T. Yun et al., "Simulation of multi-platform LiDAR for assessing total leaf area in tree crowns," Agric. For. Meteorol. 276, 107610 (2019).

19. A. M. Lefsky et al., "Surface LiDAR remote sensing of basal area and biomass in deciduous forests of eastern Maryland, USA," Remote Sens. Environ. 67(1), 83-98 (1999).

20. K. Liu et al., "The evaluation of parametric and non-parametric models for total forest biomass estimation using UAS-LiDAR," in Int. Workshop Earth Obs. Remote Sens. Appl. (2018).

21. P. Polewski et al., "Marker-free coregistration of UAV and backpack LiDAR point clouds in forested areas," ISPRS J. Photogramm. Remote Sens. 147, 307-318 (2019).

22. Q. Xu et al., "Extraction of leaf biophysical attributes based on a computer graphic-based algorithm using terrestrial laser scanning data," Remote Sens. 11(1), 15 (2019).

23. J. Bohlin, J. Wallerman, and J. E. S. Fransson, "Forest variable estimation using photogrammetric matching of digital aerial images in combination with a high-resolution DEM,' Scand. J. For. Res. 27(7), 692-699 (2012).

24. M. Messinger, G. Asner, and M. Silman, "Rapid assessments of Amazon forest structure and biomass using small unmanned aerial systems," Remote Sens. 8(8), 615 (2016).

25. L. Wallace et al., "Development of a UAV-LiDAR system with application to forest inventory," Remote Sens. 4(6), 1519-1543 (2012).

26. S. Puliti et al., "Inventory of small forest areas using an unmanned aerial system," Remote Sens. 7(8), 9632-9654 (2015).

27. V. Otero et al., "Managing mangrove forests from the sky: forest inventory using field data and unmanned aerial vehicle (UAV) imagery in the Matang Mangrove Forest Reserve, peninsular Malaysia," For. Ecol. Manage. 411, 35-45 (2018).

28. L. Cao et al., "Estimating canopy structure and biomass in bamboo forests using airborne LiDAR data," ISPRS J. Photogramm. Remote Sens. 148, 114-129 (2019).

29. L. Cao et al., "Assessment of different approaches for estimating volume change in secondary forests using bi-temporal LiDAR data," in Int. Workshop Earth Obs. Remote Sens. Appl. (2018).

30. A. R. Zahawi et al., "Using lightweight unmanned aerial vehicles to monitor tropical forest recovery," Biol. Conserv. 186, 287-295 (2015).

31. J. P. Zarco-Tejada et al., "Tree height quantification using very high resolution imagery acquired from an unmanned aerial vehicle (UAV) and automatic 3D photo-reconstruction methods," Eur. J. Agron. 55(2), 89-99 (2014).

32. B.-H. Pan, Z.-M. Zhang, and T.-R. Cao, "Natural vegetation of Yusan National Forest Park in Jiangsu Province, China," J. Cent. South Univ. For. Technol. 4, 27 (2007).

33. Z. W. Feng, X. K. Wang, and G. Wu, Biomass and Productivity of Chinese Forest Ecosystem, 1st ed., pp. 241, Science Press, Beijing, China (1999).

34. J. Ye and Z. Yang, "Biomass structure of planted Chinese fir in southern Jiangsu province, China," Acta Ecol. Sin 3, 7-14 (1983).

35. W. Qi-Ming and S. You-Guang, "A preliminary study on the biomass and production of slash pine plantation in Jiangsu Province," Acta Phytoecol. Geobot. Sin. 14(1), 1-12 (1990).

36. D. Sun, H. Ruan, and J. Ye, "Biomass structure of oak-dominated secondary forest in Kongqingshan," in Proc. Xiashu For. Ecol. Syst., China Forestry Publishing House, Beijing, China, pp. 16-22 (1992).

37. E. Næsset, "Predicting forest stand characteristics with airborne scanning laser using a practical two-stage procedure and field data," Remote Sens. Environ. 80(1), 88-99 (2002). 
38. X. Shen et al., "Prediction of forest structural parameters using airborne full-waveform LiDAR and hyperspectral data in subtropical forests," Remote Sens. 10(11), 1729 (2018).

39. S. Jayathunga, T. Owari, and S. Tsuyuki, "The use of fixed-wing UAV photogrammetry with LiDAR DTM to estimate merchantable volume and carbon stock in living biomass over a mixed conifer-broadleaf forest," Int. J. Appl. Earth Obs. Geoinf. 73, 767-777 (2018).

40. D. M. Woebbecke et al., "Color indices for weed identification under various soil, residue, and lighting conditions," in Meeting, American Society of Agricultural Engineers (1994).

41. M. Georgee and N. Joãocamargo, "Verification of color vegetation indices for automated crop imaging applications," Comput. Electron. Agric. 63(2), 282-293 (2008).

42. V. M. D'Oliveira et al., "Estimating forest biomass and identifying low-intensity logging areas using airborne scanning lidar in Antimary State Forest, Acre State, Western Brazilian Amazon," Remote Sens. Environ. 124, 479-491 (2012).

43. T. Fu et al., "Prediction of subtropical forest parameters using airborne laser scanner," J. Remote Sens. 15, 1092-1104 (2011).

44. L. Cao et al., "Mapping above- and below-ground biomass components in subtropical forests using small-footprint LiDAR," Forests 5(6), 1356-1373 (2014).

45. Q. He et al., "A study of forest parameters mapping technique using airborne LiDAR data," Adv. Earth Sci. 24(7), 748-755 (2009).

46. R. M. Lucas, A. C. Lee, and P. J. Bunting, "Retrieving forest biomass through integration of CASI and LiDAR data," Int. J. Remote Sens. 29(5), 1553-1577 (2008).

47. T. Xu et al., "Estimates of subtropical forest biomass based on airborne LiDAR and Landsat 8 OLI data," Chin. J. Plant Ecol. 39(4), 309-321 (2015).

48. S. Puliti et al., "Use of partial-coverage UAV data in sampling for large scale forest inventories," Remote Sens. Environ. 194, 115-126 (2017).

49. S. Jayathunga, T. Owari, and S. Tsuyuki, "Evaluating the performance of photogrammetric products using fixed-wing UAV imagery over a mixed conifer-broadleaf forest: comparison with airborne laser scanning," Remote Sens. 10(2), 187 (2018).

50. X. Shen et al., "Estimation of forest structural attributes using spectral indices and point clouds from UAS-based multispectral and RGB imageries," Remote Sens. 11(7), 800 (2019).

Ziqian Xu received his $\mathrm{PhD}$ in the field of ecology from Nanjing Forestry University. His research topics are ecological remote sensing, ecosystem processes, and ecosystem modeling.

Weizheng $\mathbf{L i}$ is a lecturer of Nanjing Forestry University, with research fields including UAV remote sensing.

Yuanyuan Li is a doctor of Nanjing Forestry University, with research fields including soil ecology.

Xin Shen is a doctor of Nanjing Forestry University, with research fields including silviculture.

Honghua Ruan is a professor of Nanjing Forestry University, with research fields including soil ecology and global climate change. 